\title{
Opportunities and obstacles using a clinical decision support system for maternal care in Burkina Faso
}

\author{
S Alphonse Zakane ${ }^{1,2 *}$, Lars L Gustafsson², Ali Sie ${ }^{1}$, Göran Tomson ${ }^{3,4}$, Svetla Loukanova ${ }^{5,}$, Pia \\ Bastholm-Rahmner ${ }^{4}$
}

1. Centre de Recherche en Santé de Nouna, BP 02 Nouna, Burkina Faso

2. Department of Laboratory Medicine, Division of Clinical Pharmacology, Karolinska Institutet at Karolinska University Hospital, SE-141 86, Stockholm, Sweden

3. Health Systems and Policy, Department of Public Health Sciences, Karolinska Institutet, SE17177 Stockholm, Sweden

4. Department of Learning, Informatics, Management and Ethics, Medical Management Centre (MMC), Karolinska Institutet, SE-17177 Stockholm, Sweden

5. Department of General Practice and Health Services Research, Heidelberg University Hospital, Marsilius-Arkaden, ImNeuenheimer Feld 130.3, D-69120 Heidelberg

\begin{abstract}
Objective: Maternal and neonatal mortality is high in sub-Saharan Africa. To support Healthcare Workers (HCWs), a computerized decision support system (CDSS) was piloted at six rural maternal care units in Burkina Faso. During the two years of the study period, it was apparent from reports that the CDSS was not used regularly in clinical practice. This study aimed to explore the reasons why HCWs failed to use the CDSS.

Methods: A workshop, organized as group discussions and a plenary session, was performed with 13 participants to understand their experience with the CDSS and suggest improvements if pertinent. Workshop transcripts were analyzed thematically. Socio-demographic and usage patterns of the CDSS were examined by a questionnaire and analyzed descriptively.

Results: The participants reported that the contextual basic conditions for using the CDSS were not fulfilled. These included unreliable power supply, none user-friendly partograph, the CDSS was not integrated with workflow and staff lacked motivational incentives. Despite these limitations, the HCWs reported learning benefits from guidance and alerts in the CDSS. Using the CDSS enabled them to discover problems earlier as they learned to focus on symptoms to prevent harmful situations.
\end{abstract}


Conclusion: The CDSS was not tailored to the needs and context of the users. The HCWs, defined their needs and suggested how the CDSS should be re-designed. This suggests that the successful and regular usage of any CDSS in rural settings requires the involvement of users throughout the construction and pilot-testing phases and not only during the early prototype design period.

Keywords: Burkina Faso, Computerized Clinical Decision Support System, Healthcare Workers, Maternal Care, Rural Area, Workshop

Correspondence: *S Alphonse Zakane, Centre de Recherche en Santé de Nouna, BP 02 Nouna, Burkina Faso, Department of Laboratory Medicine, Division of Clinical Pharmacology, Karolinska Institutet at Karolinska University Hospital, SE-141 86, Stockholm, Sweden. E-mail: al_zakane@yahoo.fr

DOI: 10.5210/ojphi.v9i2.7905

Copyright @2017 the author(s)

This is an Open Access article. Authors own copyright of their articles appearing in the Online Journal of Public Health Informatics. Readers may copy articles without permission of the copyright owner(s), as long as the author and OJPHI are acknowledged in the copy and the copy is used for educational, not-for-profit purposes

\section{Introduction}

In Burkina Faso, the neonatal and maternal mortality is as high as 27 per 1000 newborns and 400 per 100000 pregnancies, respectively $(1,2)$. Peripheral healthcare facilities are mainly managed by nurse and midwife assistants with occasional access to midwifes, physicians or obstetricians $(3,4)$. It is well known that skilled and educated healthcare workers (HCWs) are needed to provide high quality maternal and neonatal care (5) to help reduce neonatal and maternal mortality.

One strategy to assist HCWs in rural Africa in undertaking skilled decisions is to give easy access to guidelines and information at the "point-of-care" by using a computerized clinical decision support system (CDSS) (6,7). Unfortunately, CDSS systems are commonly designed without a clear strategy for understanding the needs of end-users and their working conditions $(3,9)$. Designing a CDSS without considering the user perspective fails to provide interest, and can raise resistance to their use in daily practice (7,9-11). The benefits of CDSS systems are ensured if they have a specific purpose, are "easy to use" and are adapted to the workflow at the healthcare facility $(3,6,7,12,13)$.

To assist HCWs in their routine work, a CDSS was designed, implemented and evaluated in six healthcare facilities in rural Burkina Faso $(3,14)$. The CDSS content was based on WHO guidelines for maternal and neonatal care $(4,14)$ with two main sections: i) a checklist ensuring that all relevant patient data are compiled and, ii) alerts and recommendations for care based on algorithms that screened entered information for any suspected pathological, clinical, or laboratory data. Furthermore, the CDSS aimed to simplify patient management and re-training in prenatal care, delivery and neonatal care. 
Before implementation of the CDSS in Burkina Faso, the HCWs at six health facilities were interviewed about their perceived needs and attitudes towards using CDSS in daily work (3). The main finding showed high expectations on the CDSS as a learning tool in maternal and neonatal care. After two years of pilot-testing, it was found that the CDSS was not used on a regular basis. Consequently, the aim of this study was to explore the reasons and explanations why the HCWs failed to use the CDSS as planned.

\section{Materials and methods}

\section{Study design}

The log-file of HCWs use of the CDSS showed that it was used in $24 \%$ of all antenatal care visits and decreased over time during the 23 months' implementation and study period (Table 1). The users were invited to an interactive workshop to discuss reasons for use or no-use of the CDSS during the study period. Such a workshop is defined as a group discussion where the participants explored their own statements in small groups and in a concluding plenary session $(15,16)$.

The study design was approved by the Nouna Institutional Review Board, Burkina Faso (Deliberation $\mathrm{N}^{\circ}$ 2010-5/CLE/CRSN) as part of the QUALMAT project (FP7-HEALTH-2007B).

Table 1: Antenatal Care (ANC) visits managed with the CDSS during the implementation and study period from June 2012 to April 2014

\begin{tabular}{lllll}
\hline & 2012 & 2013 & 2014 \\
& $($ June to Dec.) & $\begin{array}{l}\text { (Jan. to Dec.) } \\
\text { (Jan. to Apr.) }\end{array}$ & Total \\
\hline Total observations & 6536 & 10304 & 5765 & 22605 \\
\hline ANC visits managed without CDSS & $77 \%$ & $71 \%$ & $85 \%$ & $76 \%$ \\
ANC visits managed with CDSS & $23 \%$ & $29 \%$ & $15 \%$ & $24 \%$ \\
\hline
\end{tabular}

\section{Study setting and participants}

The workshop took place in Nouna health district in Burkina Faso in March 2014. The Nouna health district covers a population of 340000 inhabitants served by 43 peripheral healthcare facilities and one 80-bed district hospital.

All peripheral healthcare facilities have equal type of services: one clinic for general care, one maternity unit for family planning counseling, antenatal care, non-complicated deliveries, post delivery care and one pharmacy unit. In accordance to policies in Burkina Faso, the staff of the 
peripheral healthcare facilities includes nurses, nurse assistants, midwife assistants (except for big cities where you find midwives at this level) and a community member trained to sell drugs at the pharmacy of the facility. The six healthcare facilities (with the CDSS) were situated between 25 to $45 \mathrm{~km}$ from the district hospital. Three of the facilities had tarred roads and one facility had limited possibility to use cars (ambulance as well) for referral during rainy seasons (2 to 3 months per year).

The implementation of CDSS started in 2012 at six peripheral healthcare facilities with a total of 25 users (3). After two years, 12 users were still working in the facilities using the CDSS and all were invited to take part in the workshop. Eleven out of 12 accepted. Furthermore, two participants with background in obstetric care from the district medical team in charge of monitoring the healthcare facilities were invited to give their external view on the utilization of CDSS during the study period.

\section{Data collection}

The objectives of the workshop were to understand how the HCWs experienced the CDSS in clinical practice. The data collection had three parts:

Initially, all participants filled in a questionnaire with socio-demographic variables and their usage patterns of the CDSS.

The 13 participants (11 HCWs plus the two members from the district medical office) were divided into three groups with three to five participants from different health facilities in each group. All groups were given guiding questions (Table 2) to start the discussion. Each group was free to choose a moderator who should write a report about their answers to the questions. The group discussion lasted for 90 minutes.

Table 2. Guiding questions to capture participants' experiences of the CDSS during the group discussions

\begin{tabular}{|l|l|}
\hline Number & Question \\
\hline 1. & What is your overall impression about the CDSS? \\
\hline 2. & $\begin{array}{l}\text { What do you think about the different steps of the CDSS (data entry, guideline } \\
\text { section and training part)? }\end{array}$ \\
\hline 3. & What is your experience with the partograph? \\
\hline 4. & $\begin{array}{l}\text { What is your experience with the support equipment (laptop, solar panel and } \\
\text { batteries, etc.)? }\end{array}$ \\
\hline 5. & $\begin{array}{l}\text { Would you like to continue to use the CDSS as it is today? If yes, what is the } \\
\text { best thing with the CDSS? If no, what should be modified? }\end{array}$ \\
\hline 6. & $\begin{array}{l}\text { Have the CDSS improved your performance in clinical practice? If yes, in } \\
\text { what way? }\end{array}$ \\
\hline
\end{tabular}




\begin{tabular}{|l|l|}
\hline 7. & Imagine, how an optimal CDSS should look like according to your view? \\
\hline 8. & $\begin{array}{l}\text { What is your experience about the overall implementation, education and } \\
\text { training with the utilization of the CDSS? }\end{array}$ \\
\hline
\end{tabular}

The last part of the workshop was a plenary session lasting for two hours. Group reports were projected on a screen allowing each participant to comment. All participants were given time to talk and they were enthusiastic to express their opinions about the CDSS. Two researchers (SAZ and MK), with good knowledge of the CDSS, were in charge of the plenary session. All participants agreed that the session was audio recorded.

The participants were informed that their participation in the study was voluntary and that they could withdraw at any time, without any implications. The whole workshop took approximately four hours. No incentives were given for participants to take part apart from food and transportation from their different work places to the research center where the workshop took place.

\section{Data analysis}

\section{Questionnaire}

The answers from the individual questionnaires were entered locally by SAZ using the data-entry form of Epi Info windows version 3.5.1 (Epi Info $^{\mathrm{TM}}$, software in the public domain freely available by Centre for Disease Control (CDC), Atlanta US at www.cdc.gov/epiinfo/index.html) and analyzed using descriptive statistics (frequency and central tendency trends).

\section{b) Group discussions and c) plenary session}

The recorded material of the plenary session in French was transcribed by one researcher (SAZ) and together with the written notes from the group discussions translated into English (SAZ). All material were analyzed with inductive thematic analysis with no predetermined categories in a stepwise manner (17). The transcript, together with the written answers from the group discussions, were read several times to get a sense of the main findings.

The analysis of the transcript started by sorting all text into two main themes of strengths and weakness in relation to the use of the CDSS. The sections of text in each group were summarized and grouped by content into preliminary categories. The next step was to find related patterns within each preliminary category, which means that sections of the text were moved between categories, and new categories were formed. The categories were finally grouped under two main themes: i) inhibiting factors and ii) learning factors in relation to the use of the CDSS. Each theme is presented with its consistent category and subcategory in Table 3. Quotes were selected to illustrate each subcategory. The analysis was performed in a reciprocal way by two of the researchers SAZ (computer scientist) and PBR (behavioral scientist). 
Table 3: The final two main themes of consistent categories and subcategories

\begin{tabular}{|c|c|c|}
\hline Theme & Category & Subcategory \\
\hline \multirow[t]{5}{*}{$\begin{array}{l}\text { 1. Inhibiting } \\
\text { factors in } \\
\text { the use of } \\
\text { the CDSS }\end{array}$} & $\begin{array}{l}\text { Working environment as a } \\
\text { basic condition to use the } \\
\text { CDSS: erratic } \\
\text { maintenance and lack of } \\
\text { computers }\end{array}$ & $\begin{array}{l}\text { Hardware and software problems with } \\
\text { the computer } \\
\text { Unreliable supply of electricity } \\
\text { Shortage of computers }\end{array}$ \\
\hline & $\begin{array}{l}\text { Work process: The CDSS } \\
\text { does not fit into the } \\
\text { workflow in routine work }\end{array}$ & $\begin{array}{l}\text { The CDSS does not fit into the } \\
\text { workflow in routine work }\end{array}$ \\
\hline & $\begin{array}{l}\text { Software design: feedback } \\
\text { on statistical data for } \\
\text { performance and poor } \\
\text { user-friendliness }\end{array}$ & $\begin{array}{l}\text { Feedback of individual performance } \\
\text { data for HCWs as well as } \\
\text { statistical reports for the facility } \\
\text { Poor user-friendliness of the } \\
\text { partograph }\end{array}$ \\
\hline & $\begin{array}{l}\text { Training and knowledge: } \\
\text { lack of training in } \\
\text { maternal care and } \\
\text { computer use }\end{array}$ & $\begin{array}{l}\text { Lack of continuous training in } \\
\text { maternal care } \\
\text { Need of more training in handling } \\
\text { computers and in CDSS use }\end{array}$ \\
\hline & $\begin{array}{l}\text { Organization and } \\
\text { leadership: motivation and } \\
\text { communication }\end{array}$ & $\begin{array}{l}\text { Turnover of users and lack of } \\
\text { incentives } \\
\text { Communication and leadership }\end{array}$ \\
\hline \multirow[t]{2}{*}{$\begin{array}{l}\text { 2. Learning } \\
\text { factors in } \\
\text { the use of } \\
\text { the CDSS }\end{array}$} & $\begin{array}{l}\text { Individual learning: skills } \\
\text { in computer use and in } \\
\text { obstetrics }\end{array}$ & $\begin{array}{l}\text { Gaining skills in the usage of } \\
\text { computers } \\
\text { Learn from the CDSS registered data, } \\
\text { from alerts and incorporate } \\
\text { documentation }\end{array}$ \\
\hline & $\begin{array}{l}\text { Organizational learning: } \\
\text { improvement of quality of } \\
\text { care and eager to use } \\
\text { technology }\end{array}$ & $\begin{array}{l}\text { Improved quality in maternal care } \\
\text { A desire to experiment with new } \\
\text { technology }\end{array}$ \\
\hline
\end{tabular}

\section{Results}

The results cover (a) socio-demographic information and (b) HCW reported experience about the use of CDSS from the questionnaire, group discussion and the plenary sessions. 


\section{(a) Socio-demographic information}

Seven of the 13 participants were female and six were midwife-assistants with a mean of 7.5 years of experience in maternal care (Table 4).

Table 4. Characteristics of the participants

\begin{tabular}{|c|c|}
\hline Modality & $\begin{array}{l}\text { Number of participants } \\
n=13\end{array}$ \\
\hline \multicolumn{2}{|l|}{ Sex } \\
\hline Male & 6 \\
\hline Female & 7 \\
\hline \multicolumn{2}{|l|}{ Education } \\
\hline Primary & 3 \\
\hline Secondary First level & 5 \\
\hline $\begin{array}{l}\text { Secondary Second } \\
\text { level }\end{array}$ & $\begin{array}{l}5 \\
0\end{array}$ \\
\hline $\begin{array}{l}\text { University } \\
\text { Other }\end{array}$ & 0 \\
\hline \multicolumn{2}{|l|}{ Specialization } \\
\hline Nurse & 2 \\
\hline Midwife & 2 \\
\hline Midwife assistant & 6 \\
\hline Nurse assistant & 2 \\
\hline $\begin{array}{l}\text { Other (Nurse } \\
\text { specialized in obstetric } \\
\text { care) }\end{array}$ & 1 \\
\hline \multicolumn{2}{|l|}{ Current position } \\
\hline General consultation & 0 \\
\hline Maternity & 9 \\
\hline Vaccination & 2 \\
\hline Head of the facility & 2 \\
\hline Other & 0 \\
\hline Years of experience in & 1 to 14 years $($ Mean $=5$, Mode $=2)$ \\
\hline
\end{tabular}


current position

Years of experience in

4 to 15 years $($ Mean= 7.5 , Mode= 5) maternal cares

(b) Reported usage patterns and experience about the CDSS

All 11 participants reported that they had used the CDSS and five reported that they used it "more than once a week". However, on the follow-up question "Have you used the CDSS this week” only 1 participant responded “yes” (Table 5).

Table 5. Reported usage of the computerized Clinical Decision Support System (CDSS)

\begin{tabular}{ll}
\hline Modality & Number of participants \\
& $\mathrm{n}=11$ \\
\hline
\end{tabular}

Have you use the CDSS

Yes

\section{If use of the CDSS}

frequency

More than one a week 5

Once a week 0

Once a month 0

Sometimes 5

Infrequently 1

Have you used the CDSS

this month

$\begin{array}{ll}\text { Yes } & 2 \\ \text { No } & 9\end{array}$

\section{Have you used the CDSS}

this week

$\begin{array}{lr}\text { Yes } & 1 \\ \text { No } & 10\end{array}$


Users' experiences of the CDSS are presented under the two mains themes, inhibiting and learning factors.

1. Inhibiting factors in the use of the CDSS

1.1 Working environment as a basic condition to use the CDSS: erratic maintenance and lack of computers

\section{Hardware and software problems with the computer}

An irritation for the users was the frequent breakdown of the computers due to software bugs (standard programs of the computer as well as the CDSS software) or problems to simply start the laptop. When a computer broke down it could take up to three to four weeks to get it repaired. The participants suggested that access to a back-up computer at each facility would avoid disruption of their work.

Group1: "Provide maintenance tools like basic instructions for users and repair occasional breakdown on time when needed. Plan to include a backup computer in case one computer is broken down."

\section{Unreliable supply of electricity}

Working in remote areas without electricity makes the CDSS depending on the solar panel system for power supply. The solar panel system is sensitive and easily broke down for up to one to two months, which make the use of the CDSS uncertain. The failures of the solar panel system are essentially due to two factors: dust on the solar cells on the roofs of the facilities, or improper maintenance of the equipment's that cause break down of equipment such as voltage regulator, light bulbs or voltage convertor. The users reported that the maintenance to check the functionality of the solar power system is scheduled twice a year. They thought this is not enough.

Group1: "Sometimes the voltage convertor or voltage regulator of the solar power system broke down. We thought that maybe provision of new equipment on a regular basis like solar cell and batteries; this will reduce the risk of breakdown of the solar electricity system. Also, we suggested that the delay to repair the electricity system when breakdown occurs has to be reduced.”

\section{Shortage of computers}

As the work in the facilities often is organized in two or three teams working in different rooms, it becomes difficult with access to only one computer if this is the case.

Group 3: "When you are on duty for the maternal care, you have to perform different tasks like physical examination, vaccination or counseling for HIV testing or counseling for nutrition and delivery. These tasks are sometimes done in different rooms. Therefore, it is difficult to use the CDSS and perform all work if you are two or three employees to perform the required tasks. Because you may need to move in another room to complete one specific task ... We need two 
or three laptops per facility which are connected with the CDSS so we can use the CDSS at different place in the same facility".

1.2 Work process: The CDSS does not fit into the workflow in routine work

The HCWs complained that the CDSS doesn't fit into the workflow in routine work and causes double work for them. First, they should fill in all information on paper. This information is required by the healthcare administration for each patient (consultation card and register). Following this, they had to input the same information into the CDSS. This added approximately 30 to 40 minutes extra workload for each patient.

Group3: "Data entry takes time. It is a double work, as we have to fill in all information on paper while we already have problem with lack of staff"

Group2: "The time we spent for one patient now, before it was two patients for the same time ... now we spent 30 to 40 min for one patient in ANC, while it was 15 to 20 minutes before we had the CDSS".

1.3 Software design: Feedback on statistical data for performance and poor user-friendliness

Feedback of individual performance data for HCWs as well as statistical reports for the facility

The participants expressed a desire to have access to data from the CDSS to know how they individually performed as well as to obtain statistical reports on the performance of the facility. They wanted the statistical reports as comparisons with other facilities so they know in which area they should perform better.

Group1: "The CDSS has established an electronic document containing information collected from clinical work. We hope that this information may at any time be used for investigation purposes on maternal health quality improvement activities. At this moment, we couldn't get access to these data."

\section{Poor user-friendliness of the partograph}

The participants reported poor user-friendliness and incorrect appearance of the partograph in the CDSS, which make it less interesting to use in clinical work as it is not in accordance with present working procedures. In the CDSS, the plot of the partograph always starts at cervix opening at zero centimeter, which is incorrect in clinical practice, and this setting currently cannot be changed by the user. When the graph is not correct, it is difficult to follow the delivery process and to make a decision based on this information. The participants explained that this is one of the reasons why they rarely used the CDSS. Instead they preferred to use the paper-based partograph.

Group1: "The partograph in the CDSS does not correspond to the delivery progress in real life. When the womb is at $4 \mathrm{~cm}$ and we ought to start using the partograph, the plot shows the starting 
point at $0 \mathrm{~cm}$ that is not correct. It is not possible to start the plot at $4 \mathrm{~cm}$. This is the reason why we prefer the paper based graphics instead of the CDSS partograph.”

1.4 Training and knowledge: Lack of training in maternal care and computer use

\section{Lack of continuous training in maternal care}

The HCWs have different levels of training and experience in maternal care. Because of poor skills, some HCWs typically seek help from more experienced colleagues. This is not easy in a remote area due to distance between the facilities and the hospital, and mobile phones are generally not an alternative due to poor connectivity. A great deal of expectation was seen with the CDSS implementation as a continuous training opportunity in maternal care. Unfortunately, currently HCWs only got few retraining sessions.

Group 2: "During the intervention there was insufficient retraining in maternal care. As we have different experience on maternal care we expected to get regular retraining."

\section{Need of more training in handling computers and in CDSS usage}

Participants perceived the design of the CDSS as complex. They pointed out that they received one training session only on computer utilization and on how to use the CDSS. This was deemed as insufficient as none of HCWs taking part had worked with either computers or CDSS previously.

Group2: “The CDSS is complex to use. Sometimes we don't understand how to use the module for pregnancy delivery. When discussing the utilization of the CDSS with other colleagues from different health facilities, we have the impression of that we are using different systems (...). The CDSS should be easy to use for all workers meaning that it should be self-instructive and not complex to use."

\subsection{Organization and leadership: motivation and communication}

\section{Turnover of users and lack of incentives}

The turnover of users is high due to multiple reasons including professional promotion or family events. The respondents even reported that the CDSS has been a reason for some HCWs to change workplace.

Plenary session: "The CDSS was a motivating factor for us to stay at our posts in rural health facilities. Along the way, this has changed and some asked to move to different health facilities. The CDSS was complicated to use and there was a restriction, as we were not allowed to use the computer for other tasks. This was a real disappointment for us”.

New HCWs are not trained in how to use the CDSS, which put a pressure on senior staff to train the newly recruited staff on how the CDSS works or to do all the work by them self. 
Group3: "There was a high turnover of users, half of the users moved within one year after the beginning of the CDSS project. We received many new potential users without any training and experience to use the CDSS. It became difficult for us to train them as we have a lot to do.”

The participants reported when they started to use the CDSS they were promised different kinds of motivational triggers such as a financial incentive based on a combination of group and individual performance every six months and a non-financial incentive such as a congratulation letter, regular training on maternal care or provision of equipment for work related. These incentives were stated in a memorandum of understanding (MOU), signed by the district health officer and the country Principal Investigator of the project. The HCWs also expressed during the computer training before they started to use the CDSS and during the needs assessment study (3), their expectations to have 1) access to internet to search information and possibilities to exchange experience with others at work, and 2) possibility to use the computer for other activities than just work related activities. These expectations were not fulfilled as they had projected.

Group1: "With the project, we were expected to receive financial incentives. We were supposed to have free access to the computer for any purpose for work like reporting of facility activities or exchanging experiences with colleagues using internet or for personal tasks like watching video or social networking (Facebook). But it was only possible to use the CDSS because the computer was locked for other purposes. Furthermore, we received irregularly financial incentives based on our performance.”

\section{Communication and leadership}

The participants reported that during the course of the project implementation, they received training to follow a new procedure for the management of HIV prevention in maternal care. Still, the CDSS was not updated according to this guideline. Unless the CDSS is updated with new guidelines from the healthcare administration it is impossible for the HCWs to use the CDSS.

Group3: "We received a new version of the HIV guidelines for maternal care from the healthcare administration but it was not updated in the CDSS accordingly ... It would be good to integrate new and up to date documents related to maternal care when needed. The CDSS must be updated continuously”.

\section{Learning factors in the use of the CDSS}

Despite a range of prohibitive factors in the utilization of the CDSS, participants reported that the learning possibilities stimulated the use of the CDSS.

\subsection{Individual learning: Skills in computer use and in obstetrics}

\section{Gaining skills in the usage of computers}

The participants were pleased to have acquired computer skills by working with the CDSS. Such skills are important for them both privately and for their professional work. 
Group1: "The CDSS allow us to enhance our computer skills. We got basic computer training at the beginning of the project. And twice per month we had an IT person visiting the facilities. During these visits, we could ask questions and get answers about computers and CDSS usage. We also got this opportunity to ask the IT person for help to manage our private laptops and our private works.”

\section{Learn from the CDSS registered data, from alerts and incorporate documentation}

Participants reported that all data, from antenatal care to delivery, are stored in the CDSS. This stored information made it possible for HCWs to easily follow the progress of the pregnancy and detect problems early. This information makes it safer for mothers, especially in case of emergency.

Group 1: "The CDSS is a guide for monitoring pregnant women during antenatal care and during delivery. It also allows early detection of problems related to pregnancy and provides guidance for decision-making to deal with problems that occurs.”

Group 2: "The CDSS allows us to get early warnings of problems that may occur during pregnancy, delivery and during early first hour’s postnatal care.”

Participants reported satisfaction with the guidance of the CDSS. They reported that the CDSS help them in rapid diagnosis and safe treatment of the mother. When the HCWs get access to the registered information and alerts from the CDSS they got to learn "what symptoms to look for in their patient" which help them to discover problems earlier. Furthermore, the users consider that with the CDSS they get guidance on answers to routine questions that earlier needed consultations with colleagues.

Group2: "The CDSS creates efficiency in the management of patients, for example the prescribing is scientifically based and not by imitation of the actions by colleagues. We understand what we are doing before making the prescription. The CDSS guide us through the diagnosis to prescription.”

\subsection{Organizational learning: improvement of quality of care and eager to use technology}

\section{Improved quality in maternal care}

The use of the CDSS has improved the care as reported by the two participants from the healthcare administration. The users confirm this statement by expressing that they feel safer and secure in their work when making decisions.

Group2: “The CDSS improved considerably our knowledge in obstetric care: no matter of our profile at the beginning of the project. We all gained skills and we are now comfortable to provide ANC or delivery without doubt”

Plenary session (Member of the district management team): "From what I heard and what I have seen, the healthcare facilities under the CDSS project were well organized and performed well. The maternal and neonatal care indicators are good in these healthcare facilities, I mean 
mortality and morbidity. The CDSS made HCWs more knowledgeable in maternal care. By extending access of the CDSS to all healthcare facilities, this will improve maternal care indicators by reducing maternal death, stillbirth and neonatal mortality. This will also reduce delay for referral to hospital in case of complication during pregnancy.”

\section{A desire to experiment with new technology}

The participants expressed a desire to be able to contact colleagues and experts internationally by e-mail and/or video through the CDSS. This would be an opportunity to learn more.

Group1: "The idea is to have the CDSS and new technique involving the possibility to have video discussion, email or real-time discussion with experts or specialist on maternal care."

\section{Discussion}

In the beginning of the implementation of the CDSS, the HCWs were enthusiastic to use the system (3). However, when the project proceeded they reduced their use. Our study revealed several reasons that inhibited the use of the CDSS. The technical challenges included unreliable power supply, a non-user friendly partograph, the poor integration of the CDSS into the workflow leading to high workload, and finally, the failure to adhere to promised motivational incentives combined with the high turn-over of users, didn't facilitate the use of the CDSS. Despite these obstacles, the HCWs reported opportunities by learning moments from using the CDSS in both maternal care and in computer use.

There are several technical challenges with the CDSS when using it in remote healthcare facilities in Africa. To prevent problems with unreliable power supply or computer literacy, all facilities implementing the CDSS received a solar panel system to support reliable power supply. Nevertheless, the participants in the workshop reported poor maintenance support and unreliable electricity supply, i.e. prime conditions necessary to be able to use the CDSS. This was even identified as an important factor to be considered by the participants in interviews before they started to use the CDSS (3). The problem with reliable electricity supply is well-known and was reported as a basic need in a study from Malawi $(18,19)$ where they maintained successful use of the system by access to a low voltage computer. In addition, a review of ICT studies from Africa, it was stated that functional solar panels for power supply can ensure reliable access to ICTsystems in rural settings (20). Using the same CDSS-systems as in Burkina Faso, the Tanzanian users reported similar electricity challenges, while there was no distractions in Ghana since they had access to the national grid electricity system (21).

Another technical factor inhibiting the use of the CDSS were the design of the partograph. The partograph is a critical decision tool for HCWs to follow the course of pregnancy deliveries graphically to know when to intervene (22). The HCWs did not use the partograph in the CDSS as the graph was not designed according to recommendations (22). When the graphical system does not cover clinical variation in the width of cervix, it is difficult for the HCWs to observe the delivery process and make a decision from the information. The participants explained that this is one of the reasons why they rarely used the CDSS. Instead they preferred to use the paperbased partograph. 
In Burkina Faso, all respondents reported that the CDSS was not well integrated into the clinical workflow, which also caused limited use of the CDSS. The consequence of this is the double documentation of patient data where the HCWs had to document all patient information both into the CDSS and onto the paper-based medical record. This is time consuming and increases the workload. To overcome the problem with double documentation, one suggestion is to integrate the CDSS with the patient medical record as earlier research (23) has showed that HCWs are keen to accept and use technologies such as a CDSS which follows their working process $(12,24)$. Furthermore, a practical issue that takes time and disturbs their workflow is that they only have access to one computer per facility. This is a concern as HCWs typically change room to complete the ANC or to perform a delivery. This creates unwillingness to use the CDSS due to extra efforts this may need. Indeed using the same CDSS, HCWs in Ghana and Tanzania reported that the use of the CDSS multiplied the time spent for ANC by 2.6 in Ghana (going from 7.4 minutes to 19.2 minutes) and 1.7 in Tanzania (going from 15.1 minutes to 25.5 minutes) (13). This is in line with our findings in Burkina Faso where the HCWs reported the time spent for ANC is 15 minutes more with the CDSS. Likewise, a recent study concluded that the usability problem of the CDSS can be overcome if the designers increased the link of the CDSS with the existing working process [24].

One important factor for the HCWs was that they have been promised to receive different kinds of motivational triggers (financial and non-financial incentives) to use the CDSS. They expected to have access to Internet to search for information and to use the computer for other than just work related purposes. In practice, the HCWs got irregularly financial incentives but had no possibility to use the computer for other purposes than work related questions. This led to decrease their motivation to use the CDSS. The context of Ghana showed that retracted incentives together with a high workload led to less motivation and a high turnover of HCWs (25). This is in line with results from this study where the HCWs reported that lack of incentives together with the increased workload with the CDSS partly caused turnover of HCWs.

Despite flaws and weak points during the implementation process of the CDSS, the HCWs were eager to learn more and optimize maternal care by using the CDSS. The respondents reported that they learnt from the guidance and alerts in the CDSS, thereby learning what symptoms to look for in their patients. This information provided by the CDSS supports them in discovering problems earlier. With this information, the HCWs feel more confident in their decisions regarding ANC. Furthermore; the HCWs considered that they get guidance for routine questions through using the CDSS where they usually needed colleagues' advice. This is in concordance with the users from Ghana and Tanzania, who also reported that they have learnt by using the CDSS $(13,21)$. In Ghana (13), the HCWs were not asking for information about patients' history before they started to use the CDSS, but started to do so after implementation of the CDSS according to WHO and National health authorities (13). When we know that the conducting ANC can prevent complications in pregnancy and detect early the possible need for referral of the mother to a higher level of care such as a hospital (26), the findings from this study showed that the use of a CDSS can support HCWs to give skilled care.

There were three prototypes of the CDSS before the final software was available. The first prototype was demonstrated to HCWs with low computer literacy in Burkina Faso, Ghana and Tanzania in order to have their feedback. Later, the two other versions of the prototype 
respectively were used to train the HCWs and to be used as a test by HCWs to obtain reported feedback on the errors or bugs. All feedback reports were used to develop the final CDSS. Meanwhile, before the HCWs started testing the last prototype, a study was conducted in Burkina Faso to capture the needs and attitudes of HCWs towards accessing guidelines for maternal and neonatal care through CDSS (3). Unfortunately, the results from this study were delayed and the final version of the CDSS was not based on this information. This may have done that the users perceived the CDSS as an expert-driven software (built according to researchers or experts) and not from their point of view. The process to enhance the usefulness of the CDSS is to integrate the interest of the end-users along the designing process (24). However, it is not enough to focus on the users' involvement in the prototyping. To obtain better acceptance from the end-user, it is necessary to ask for their needs in depth (analysis of the context and actual working environment), involve end-users feedback during the design process (programming of the different prototype) and then continuously evaluate the implemented CDSS in practice (24).

\section{Methodological considerations and limitations}

A workshop was preferred to individual interviews as the workshop methodology promoted group interaction. In group discussions, informants can help each other to explore and clarify their views, which would less easily be possible in individual interviews (26).To facilitate the plenary discussions, and to allow all respondents to express their opinions, we started with small group discussions. In this setting, the respondents were familiar with each other which might have made them avoid talking about important issues that negatively could affect their relations (27). Regarding the results of this study, with the critical opinion the HCWs have on the usefulness of the CDSS in clinical practice, we believe that the familiarity was one of the strengths of the workshop. Furthermore, we observed that the discussion during the plenary session was open-minded and should favor open discussions and sharing of experiences.

Another group dynamic issue to consider was to include two people from the health care administration office to attend the workshop. Their participation may have restricted HCW feedback. However, in the analysis of the data with this question in mind, we could not find any differences between the groups including an employee from the healthcare administration office and the one without an employee from the healthcare administration office. When we want to investigate the attitudes of a large group of users towards a new technology such as a CDSS, another method such as a questionnaire with quantitative measurements may be preferred $(28,29)$. One of the most used methods in these cases are based on the Technology Acceptance Model (TAM) for examining key factors for easiness of use and the perceived usefulness (28). In this study, we used the data collection method with a workshop instead of using the TAMquestionnaire because of the small number of participants. Since this study is a qualitative study, the findings cannot be generalized. However, the findings might well be transferable to similar contexts and settings. We found in our previous study (3) that both well-trained staff members (nurses and midwives) as well as assistants (nurses and midwives) had joint interests to use a new sophisticated technique such as a CDSS and to be reminded about best procedures for optimal maternal care. In addition, they could clearly describe how a CDSS should be designed and introduced in rural settings in an interactive workshop. The first aspect of what were reported is close to the "usefulness" perspective and the second is close to the "easiness of use" 
perspective. This supports the view that an interactive stepwise workshop, combining group and plenary sessions, may help define similar factors that are summarized with the TAM model. The present findings should therefore be relevant when developing CDSS in remote rural areas.

\section{Conclusion}

HCWs performance in rural settings in Africa depends on motivational issues and contextual factors such as working conditions to retain them. In Burkina Faso, HCWs were keen to use the CDSS at the beginning of the project. However, in time this enthusiastic expectation turned to demotivation. This lack of motivation was due to many factors including lack of perceived incentives and none integration of the CDSS into the workflow which appreciably increased the workload. Technical problems such as unreliable power supply and incorrect partograph also inhibited the regular use of the CDSS. Despite these deterrents, HCWs reported that they have learned in implementation of standards in maternal care provision and the usage of computers. To implement a CDSS in a rural area remains challenging but can be overcome if end-users' needs are put in perspective from the very beginning and during the different stages of the construction and testing the CDSS.

\section{Authors' contribution}

SAZ, PBR, and LLG contributed to the conception and design of the study and to interpretation of the data. SAZ conducted the workshop and analyzed the data in a reciprocal way with PBR and with quality controls and inputs from LLG.

SAZ, PBR, LLG, AS, and GT outlined the disposition of the manuscript. The first draft was written by SAZ and PBR and completed by PBR, SAZ and LLG with inputs by AS, GT and SL. The final manuscript was circulated among all authors that approved the final manuscript.

\section{Competing Interests}

No Competing Interests

\section{Acknowledgements}

This work was supported by European Union through the grants 22982 and 654237 from the $7^{\text {th }}$ EU Framework Program.

This study was performed in Burkina Faso and nested into the QUALMAT (Quality of prenatal and maternal care: bridging the know-do gap) (www.qualmat.net) The project includes the development and implementation of a system of performance based incentives and a computer- 
assisted clinical decision support system (CDSS) based on WHO guidelines for maternal and neonatal care $(4,14)$. The CDSS intervention was carried out in Burkina Faso, in Ghana and in Tanzania $(3,13,14,21)$ with two main sections $(13)$ : a checklist ensuring that all relevant data are carried out and algorithms that screening the entered data for pathological issue and give immediate alerts or comprehensive recommendations.

The authors thanks to Moubassira Kagone (MK) for his help to moderate the workshop and to Victor Coulibaly for technical support and to Brian Godman for language review of the manuscript.

Thanks to the participants of the QUALMAT health facilities for their participation in this workshop for scale-up the future implementation of CDSS in remote area Africa.

\section{References}

1. WHO. UNICEF, UNFPA and the World Bank estimates. Trends in maternal mortality: 1990 to 2013. [cited 2017 Apr 10]. Available from: http://apps.who.int/iris/bitstream/10665/112682/2/9789241507226_eng.pdf?ua=1

2. UNICEF/WHO/The World Bank/UN Pop Div. Levels and Trends in Child Mortality. Report 2014. [cited 2017 Apr 10]. Available from:

https://www.unicef.org/media/files/Levels_and_Trends_in_Child_Mortality_2014.pdf

3. Zakane SA, Gustafsson LL, Tomson G, Loukanova S, Sié A, et al. 2014. Guidelines for maternal and neonatal "point of care": Needs of and attitudes towards a computerized clinical decision support system in rural Burkina Faso. Int J Med Inform. 83(6), 459-69.

PubMedhttps://doi.org/10.1016/j.ijmedinf.2014.01.013

4. Baker U, Tomson G, Somé M, Kouyaté B, Williams J, et al."How to know what you need to do": a cross-country comparison of maternal health guidelines in Burkina Faso, Ghana and Tanzania. 2012. Implement Sci IS. 13;7:31.

5. WHO report 2003. [cited 2017 Apr 10]. Available from: http://www.who.int/whr/2003/en/whr03_en.pdf?ua=1

6. Nilseng J, Gustafsson LL, Nungu A, Bastholm-Rahmner P, Mazali D, et al. A cross-sectional pilot study assessing needs and attitudes to implementation of Information and Communication Technology for rational use of medicines among healthcare staff in rural Tanzania. 2014. BMC Med Inform Decis Mak.14(1), 1. PubMedhttps://doi.org/10.1186/1472-6947-14-78

7. Chattopadhyay S. 2010. A framework for studying perceptions of rural healthcare staff and basic ICT support for e-health use: an Indian experience. Telemed E-Health.16(1), 80-88. PubMedhttps://doi.org/10.1089/tmj.2009.0081 
8. Machingura PI, Adekola O, Mueni E, Oaiya O, Gustafsson LL, et al. 2014. Perceived value of applying Information Communication Technology to implement guidelines in developing countries; an online questionnaire study among public health workers. Online J Public Health Inform. 6(2). PubMedhttps://doi.org/10.5210/ojphi.v6i2.5368

9. Kaplan B. 2001. Evaluating informatics applications - clinical decision support systems literature review. Int J Med Inform. 64(1), 15-37. PubMedhttps://doi.org/10.1016/S1386-5056(01)00183-6

10. Ammenwerth E, Nykänen P, Rigby M, de Keizer N. 2013. Clinical decision support systems: Need for evidence, need for evaluation. ArtifIntell Med. 59(1), 1-3. PubMedhttps://doi.org/10.1016/j.artmed.2013.05.001

11. Carroll C, Marsden P, Soden P, Naylor E, New J, et al. 2002. Involving users in the design and usability evaluation of a clinical decision support system. Comput Methods Programs Biomed. 69(2), 123-35. PubMedhttps://doi.org/10.1016/S0169-2607(02)00036-6

12. Jimoh L, Pate MA, Lin L, Schulman KA. 2012. A model for the adoption of ICT by health workers in Africa. Int J Med Inform. 81(11), 773-81. PubMedhttps://doi.org/10.1016/j.ijmedinf.2012.08.005

13. Mensah N, Sukums F, Awine T, Meid A, Williams J, et al. 2015. Impact of an electronic clinical decision support system on workflow in antenatal care: the QUALMAT eCDSS in rural health care facilities in Ghana and Tanzania. Glob Health Action. 8(0).

14. Blank A, Prytherch H, Kaltschmidt J, Krings A, Sukums F, et al. 2013. "Quality of prenatal and maternal care: bridging the know-do gap”(QUALMAT study): an electronic clinical decision support system for rural Sub-Saharan Africa. BMC Med InformDecisMak. 13(1), 1. PubMedhttps://doi.org/10.1186/1472-6947-13-44

15. Corn M, Rudzinski KA, Cahn MA. 2002. Bridging the Gap in Medical Informatics and Health Services Research: Workshop Results and Next Steps. J Am Med Inform Assoc. 9(2), •••. PubMedhttps://doi.org/10.1197/jamia.M0971

16. Coleman JJ, van der Sijs H, Haefeli WE, Slight SP, McDowell SE, et al. On the alert: future priorities for alerts in clinical decision support for computerized physician order entry identified from a European workshop. 2013. BMC Med Inform DecisMak. 13(1), 111.

17. Patton MQ. 2002. Qualitative Research \& Evaluation Methods. Sage.

18. Gadabu OJ, Manjomo RC, Mwakilama SG, Douglas GP, Harries AD, et al. 2014. An electronic register for vital registration in a rural village with no electricity in Malawi. Public Health Action. 4(3), 145-49. PubMedhttps://doi.org/10.5588/pha.14.0015

19. Douglas GP, Deula RA, Connor SE. 2003. The Lilongwe Central Hospital Patient Management Information System: A Success in Computer-Based Order Entry Where One Might Least Expect It. AMIA AnnuSymp Proc., 833. PubMed 
20. Lucas H. 2008. Information and communications technology for future health systems in developing countries. Soc Sci Med. 66(10), 2122-32.

PubMedhttps://doi.org/10.1016/j.socscimed.2008.01.033

21. Sukums F, Mensah N, Mpembeni R, Massawe S, Duysburgh E, et al. 2015. Promising adoption of an electronic clinical decision support system for antenatal and intrapartum care in rural primary healthcare facilities in sub-Saharan Africa: The QUALMAT experience. Int J Med Inform. 84(9), 647-57. PubMedhttps://doi.org/10.1016/j.ijmedinf.2015.05.002

22. Yisma E, Dessalegn B, Astatkie A, Fesseha N. 2013. Completion of the modified World Health Organization (WHO) partograph during labour in public health institutions of Addis Ababa, Ethiopia. Reprod Health. 10(1), 1. PubMedhttps://doi.org/10.1186/1742-4755-10-23

23. Sambasivan M, Esmaeilzadeh P, Kumar N, Nezakati H. 2012. Intention to adopt clinical decision support systems in a developing country: effect of Physician's perceived professional autonomy, involvement and belief: a cross-sectional study. BMC Med Inform DecisMak. 12, 142.

PubMedhttps://doi.org/10.1186/1472-6947-12-142

24. Kilsdonk E, Peute LW, Riezebos RJ, Kremer LC, Jaspers MWM. 2013. From an expert-driven paper guideline to a user-centred decision support system: A usability comparison study. Artifintell Med. 59(1), 5-13. PubMedhttps://doi.org/10.1016/j.artmed.2013.04.004

25. Bonenberger M, Aikins M, Akweongo P, Wyss K. 2014. The effects of health worker motivation and job satisfaction on turnover intention in Ghana: a cross-sectional study. Hum Resour Health. 12(1), 1. PubMedhttps://doi.org/10.1186/1478-4491-12-43

26. Kitzinger J. Qualitative Research: Introducing focus groups. 1995. BMJ. 29;311(7000):299_ 302.

27. Dahlgren L. Qualitative methodology for international public health. 2. ed. Umeå: Epidemiology and Public Health Sciences, Departement of Public Health and Clinical Medicine, Umeå University; 2007.

28. Holden RJ, Karsh B-T. 2010. The Technology Acceptance Model: Its past and its future in health care. $J$ Biomed Inform. 43(1), 159-72. PubMedhttps://doi.org/10.1016/j.jbi.2009.07.002

29. Venkatesh V, Morris M, Davis G. 2003. User acceptance of information technology: toward a unified view. Manage InfSyst Q. 27, 425-78. 